Article

\title{
Unidirectional Slow Light Transmission in Heterostructure Photonic Crystal Waveguide
}

\author{
Qiuyue Zhang ${ }^{1} \mathbb{D}$ and Xun $\mathrm{Li}^{1,2, *}$ \\ 1 Wuhan National Laboratory for Optoelectronics, Huazhong University of Science and Technology, \\ Wuhan 430074, China; zhangqiuyuevip2011@163.com \\ 2 Department of Electrical and Computer Engineering, McMaster University, Hamilton, ON L8S 4K1, Canada \\ * Correspondence: lixun@mcmaster.ca; Tel.: +1-905-525-9140 (ext. 27698)
}

Received: 9 September 2018; Accepted: 7 October 2018; Published: 9 October 2018

Featured Application: The proposed unidirectional backscattering-immune device may provide a potential way to realize robust optical delay lines and robust on-chip optical buffers.

\begin{abstract}
In conventional photonic crystal systems, extrinsic scattering resulting from random manufacturing defects or environmental changes is a major source of loss that causes performance degradation, and the backscattering loss is amplified as the group velocity slows down. In order to overcome the limitations in slow light systems, we propose a backscattering-immune slow light waveguide design. The waveguide is based on an interface between a square lattice of magneto-optical photonic crystal with precisely tailored rod radii of the first two rows and a titled 45 degrees square lattice of Alumina photonic crystal with an aligned band gap. High group indices of $77,68,64$, and 60 with the normalized frequency bandwidths of $0.444 \%, 0.481 \%, 0.485 \%$, and $0.491 \%$ are obtained, respectively. The corresponding normalized delay-bandwidth products remain around 0.32 for all cases, which are higher than previously reported works based on rod radius adjustment. The robustness for the edge modes against different types of interfacial defects is observed for the lack of backward propagation modes at the same frequencies as the unidirectional edge modes. Furthermore, the transmission direction can be controlled by the sign of the externally applied magnetic field normal to the plane.
\end{abstract}

Keywords: delay-bandwidth products; group velocity dispersion; flat band; slow light; interfacial defects; backscattering-immune

\section{Introduction}

The velocity of light in vacuum is approximately $2.99 \times 10^{8} \mathrm{~m} / \mathrm{s}$, fast enough to circle the Earth once in $0.3 \mathrm{~s}$. However, faster is not always better, such an ultrahigh speed makes it very difficult to control the optical signals in time domain [1,2]. Slow light with a much slower group velocity makes it possible to delay and temporarily store light in all-optical systems [3-6]. Among all the approaches to realize slow light, photonic crystal waveguide (PCW) has drawn much attention as it supports room temperature operation and is compatible with on-chip integration [7-16]. However, the performance of the conventional photonic crystal (PC) systems is very vulnerable to the backscattering loss induced by disorders or defects $[17,18]$. The backscattering loss is amplified as the group velocity slows down in the waveguide $[19,20]$.

The development of the photonic chiral edge state (CES) [21-24] may provide an approach to overcome the limitations related to backscattering loss in slow light systems, as it is robust against scattering from interfacial defects. The existence of the photonic CESs at the interface of a two-dimensional (2D) magneto-optical photonic crystal (MOPC) was first theoretically proposed by 
Haldane $[25,26]$ and was then experimentally verified by Wang et al. $[27,28]$ in microwave regime. Since the CESs are leaky in the free space [21,29], a regular PC with an aligned band gap has to be bounded to the interface of the MOPC to prevent the radiation leakage. The constructed waveguide only supports unidirectional modes as it has only positive (negative) group velocities and the backscattering is completely suppressed. This enabled the light transmission to be immune to fabrication imperfections. The unidirectional transmission feature is of great significance for slow light systems and may provide robust designs for optical delay and light storage applications [30].

There are various approaches to tailor the dispersion curves to realize slow light in PC systems: modification the radii of air holes [31], shifting the air holes to specific directions [32], infiltrating optical fluid into the air holes [33], infiltrating microfluidic selectively [34,35], using asymmetric engineering structure [36], merging coupled cavities [37] and, introducing alternative rows of ellipse-holes [38]. In all the above mentioned ways, adjusting the rod radius is relatively easy to control and have less fabrication issues.

In this paper, we propose a unidirectional backscattering-immune slow light waveguide. The waveguide is constructed by a square lattice of MOPC with the rod radii of the first two rows adjacent to the waveguide core are precisely tailored and a titled $45^{\circ}$ square lattice of Alumina PC as an ancillary cladding. A flat band for slow light is obtained and the slope (group velocities) of the waveguide dispersion curves process only positive (or only negative) values, which is determined by the sign of the externally applied magnetic field. In the waveguide, the backscattering is completely suppressed for the lack of the backward transmission channel. This unique feature ensures that the design can avoid performance degradation induced by impurities or defects, which is difficult to realize in conventional slow light systems.

The remaining content of this work is arranged as follows: to better understand the content of the subsequent sections, we briefly introduce the theoretical background of slow light in Section 2. In Section 3, we firstly introduce the design of the unidirectional slow light waveguide and then exhibit the slow light characteristics and the backscattering-immune properties of the waveguide in detail. The followed simulation results are based on the finite element method (FEM).

\section{Theoretical Background}

The group velocity $v_{g}$ (or equivalently the group index $n_{g}=c / v_{g}, c$ denotes the speed of light in vacuum) is not the only key parameter to evaluate the performance of slow light devices. We should also take into account the frequency bandwidth, group velocity dispersion (GVD), and the propagation loss. The frequency bandwidth and the group index are encapsulated by the normalized delay-bandwidth product (NDBP) [16,39]. The NDBP indicates the obtained compromise between a large operating bandwidth and a large group index, as given by

$$
\mathrm{NDBP}=\widetilde{n}_{g} \times \frac{\Delta \omega}{\omega_{0}}
$$

$\widetilde{n}_{g}=\int_{\omega_{0}}^{\omega_{0}+\Delta \omega} n_{g}(\omega) \frac{\mathrm{d} \omega}{\Delta \omega}$ is an average group index in the desired slow light bandwidth. $\Delta \omega / \omega_{0}$ denotes the normalized frequency bandwidth, where $\Delta \omega$ represents the difference between the maximum and the minimum frequency in the slow light region and $\omega_{0}$ represents the center frequency. The reduction of $v_{g}$ should be balanced with the bandwidth requirements in practical applications. The frequency bandwidth is considered as the frequency range corresponding to a $\pm 10 \%$ group index variation with respect to the obtained mean $\widetilde{n}_{g}$ value $[16,39,40]$.

The group velocity is strongly dependent on the operation frequencies [41,42], as quantified by the second-order derivative of the dispersion relation:

$$
\beta_{2}=\frac{\mathrm{d}^{2} k}{\mathrm{~d} \omega^{2}}=\frac{1}{c} \frac{\mathrm{d} n_{g}}{\mathrm{~d} \omega}
$$


$\beta_{2}$ is the GVD parameter. Large $\beta_{2}$ can lead to pulse distortion, and thereby degrades the performance of the slow light device. Taking the practical application requirements into consideration, the GVD parameter should below the order of $10^{6}\left(\frac{a}{2 \pi c^{2}}\right)[43,44]$ ( $a$ is the lattice constant in PC systems).

The propagation loss, mainly due to light scattering, is the most critical limitations to be solved in slow light systems. The scattering loss is mainly due to random fabrication imperfections or environmental changes. It increases quadratically with the reduced $v_{g}$ as proposed theoretically in [19] and certified experimentally in [20]. If the propagation loss penalty is too high, the superiority of slow light might be compromised. To overcome this crucial limitation in slow light systems, we propose a backscattering-immune slow light waveguide as demonstrated in the followed sections.

\section{Model Design and Simulation Results}

\subsection{The Unidirectinal Waveguide and the Edge Modes}

Our proposed design is based on an interface between two types of PC structures and the schematic configuration is depicted in the inset in Figure 1. The lower half plane is a square lattice of MOPC consisting of Yttrium-Iron-Garnet (YIG) rods $\left(\varepsilon=15 \varepsilon_{0}, r=0.11 a\right)$ [28], where $a=38.69 \mathrm{~mm}$ is the lattice constant. With an externally applied magnetic field $H_{0}=1.27 \times 10^{5} \mathrm{~A} / \mathrm{m}$ normal to the plane (along the $+z$ direction), the induced magnetic permeability sensor is given as [45]

$$
\begin{gathered}
\mu=\left[\begin{array}{ccc}
\mu_{r} & i \mu_{k} & 0 \\
-i \mu_{k} & \mu_{r} & 0 \\
0 & 0 & 1
\end{array}\right] \mu_{0} \\
\mu_{r}=1+\frac{\omega_{m} \omega_{0}}{\omega_{0}^{2}-\omega^{2}}, \mu_{k}=\frac{\omega_{m} \omega}{\omega_{0}^{2}-\omega^{2}} .
\end{gathered}
$$

Here $\omega, \omega_{0}=\mu_{0} \gamma H_{0}$ and $\omega_{m}=\mu_{0} \gamma M_{s}$ indicate the operating frequency of the microwave field, the procession resonance frequency and the characteristic frequency, respectively $\left(\gamma=1.759 \times 10^{11} \mathrm{C} / \mathrm{kg}\right.$ is the gyromagnetic ratio). The saturation magnetization of the YIG rods is $M_{s}=1.42 \times 10^{5} \mathrm{~A} / \mathrm{m}$. If the external magnetic field applied along $-z$ direction, both $\omega_{0}$ and $\omega_{m}$ change signs accordingly. According to Equation (4), it can be inferred that $\mu_{r}$ remains unchanged, but $\mu_{k}$ becomes its opposite value. As the edge modes at the interface of the MOPC exist inside the light cone and thus will be leaky in the free space. A titled $45^{\circ}$ square lattice of Alumina PC (the upper half plane) with an aligned band gap $\left(\varepsilon=10 \varepsilon_{0}, R=0.115 a\right)$ has to be attached to the interface of the MOPC. Therefore, the excited edges are evanescent in both two types of PC structures.

The dashed rectangle in the inset in Figure 1 indicates a supercell of the waveguide. Using a finite element eigenfrequency analysis and imposing period boundary conditions at the supercell's borders, we can get the band diagram of the waveguide. Theoretically, the supercell must contain infinite rods in $y$ direction, while a supercell of $10 \times 1$ unite cells of the waveguide can satisfy the accuracy requirements in practical calculations. With an externally applied magnetic field along the $+z$ direction, the projected band diagram of the unidirectional waveguide for transverse magnetic (TM) modes (electric field is polarized along $z$-axis) are given in Figure 1. The shadow regions are the bulk modes of full MOPC and Alumina PC and the red curve denotes the unidirectional edge mode. As shown in Figure 1, the slope of the red curve (group velocities) has only positive values. It indicates that the waveguide support only forward transmission and the opposite propagation is completely prohibited. This unique feature insures that the edge modes are robust against interfacial disorders or defects. It is of significant importance for slow light systems. 


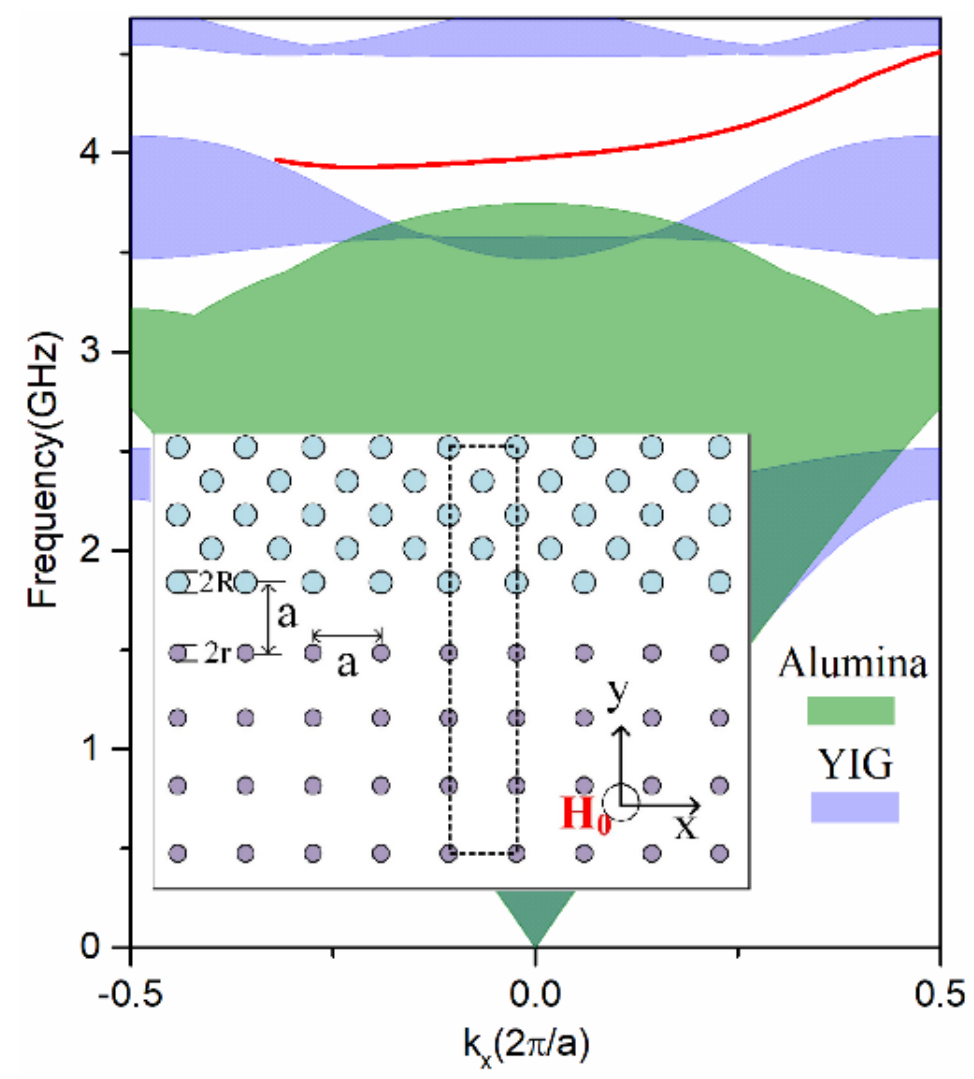

Figure 1. The projected band diagram of the waveguide. The corresponding geometry configuration is illustrated in the inset, where the lower plane is the magneto-optical photonic crystal (MOPC) $\left(\varepsilon=15 \varepsilon_{0}, r=0.11 a\right)$, the upper plane is the Alumina photonic crystal (PC) $\left(\varepsilon=10 \varepsilon_{0}, R=0.115 a\right)$, the dashed rectangle indicates a supercell of the waveguide. The red curve is the edge mode at the interface of the MOPC.

\subsection{Slow Light Properties}

Since the first few rows of YIG rods have the greatest influence on the shape of the dispersion curve, we adjust the radii of the YIG rods to obtain a flat band region for slow light (a large NDBP value and a low GVD value) with the unidirectional feature. The new configuration of the waveguide is shown in Figure 2, the radii of the YIG rods in the first and second rows adjacent to the waveguide core are denoted by $r_{1}$ and $r_{2}$, respectively. We perform a serious of numerical simulations for different $r_{1}$ values and find that when $r_{1}$ goes beyond the range of $0.075 a$ to $0.08 a$, the unique unidirectionality of the edge modes vanishes. Therefore, we present the dispersion diagram for parameters $r_{1}=0.075 a$, $r_{1}=0.077 a, r_{1}=0.08 a$, and $r_{1}=0.11 a$, as shown in Figure 3a. The corresponding group index characteristics are illustrated in Figure $3 \mathrm{~b}$. The dispersion curve becomes flat at $r_{1}=0.077 a$ and a step-like region (corresponding to the slow light region) appears, which indicates a low GVD feature. In the cases of $r_{1}=0.075 a$ and $r_{1}=0.08 a$, the curve gets very steep and the step-like behavior disappears. 


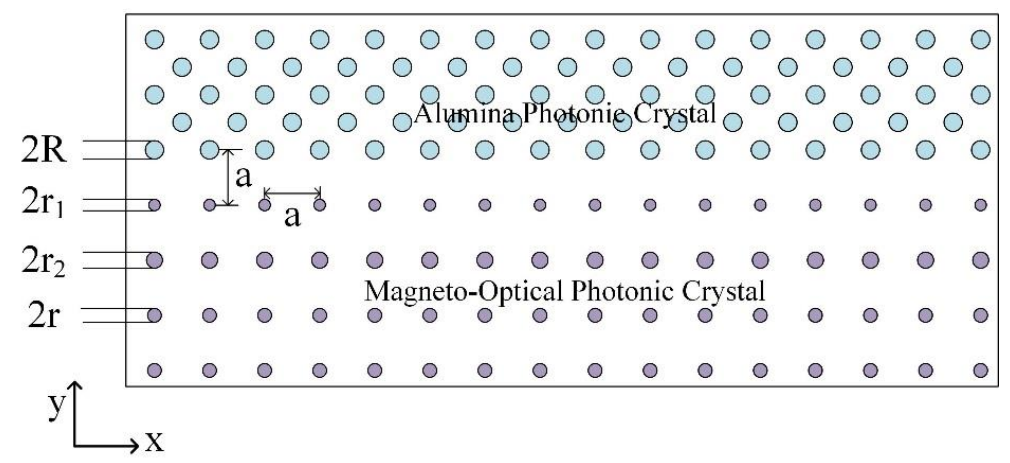

Figure 2. The geometry configuration of the one-way slow light photonic crystal waveguide (PCW). The upper half plane is still a titled $45^{\circ}$ square lattice of Alumina PC as an ancillary cladding, and the lower half plane is a square lattice of MOPC with the rod radii of first two rows are precisely tuned. The radii of the Yttrium-Iron-Garnet (YIG) rods in the first and the second rows adjacent to the waveguide are given by $r_{1}$ and $r_{2}$, respectively.

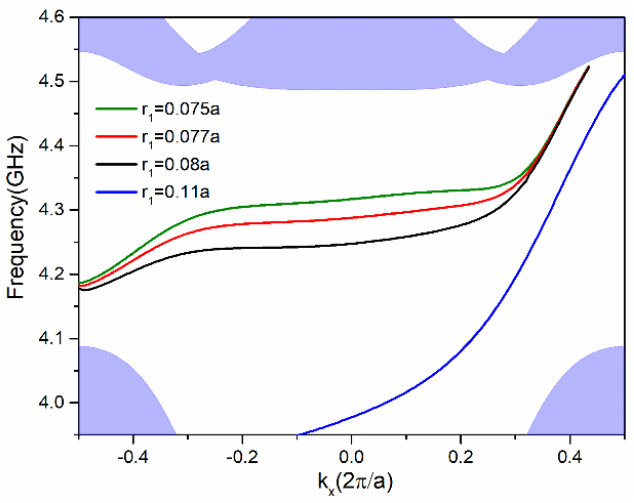

(a)

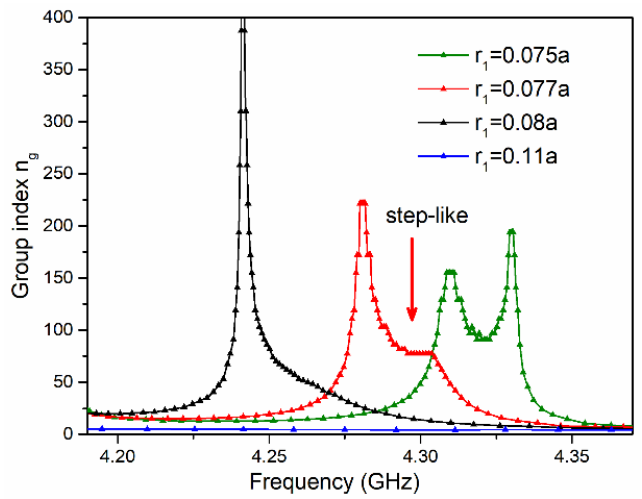

(b)

Figure 3. (a) Dispersion curves of the edge modes for different $r_{1}$ with an externally applied magnetic field along the $+z$ direction. The shadow region denotes the extended modes of a perfect MOPC; (b) Group indices as a function of the frequencies, the relevant step-like behavior is indicated.

Based on the optimum $r_{1}$, we then adjust $r_{2}$ to further engineer the dispersion curve. Figure $4 \mathrm{a}$ illustrates the projected band diagram of TM modes with an external magnetic field applied in the $+z$ direction for parameters $r_{1}=0.077 a, r_{2}=0.113 a ; r_{1}=0.078 a, r_{2}=0.115 a ; r_{1}=0.079 a, r_{2}=0.117 a$; and $r_{1}=0.08 a, r_{2}=0.118 a$. The slope of the curves (group velocities) have only positive values and the flat band regions for slow light are highlighted by the thick solid lines. The corresponding frequency range of the slow light region are $4.2886 \mathrm{GHz}-4.3077 \mathrm{GHz}, 4.2744 \mathrm{GHz}-4.295 \mathrm{GHz}, 4.2599 \mathrm{GHz}-4.2806$ $\mathrm{GHz}$, and 4.2483 GHz-4.2692 GHz. The thick solid lines are approximate linear, which correspond to the step-like regions in Figure 5a. As illustrated in Figure 5a, the step-like behavior with a small change in group index in a relatively large frequency bandwidth is found in all cases, which indicates a large NDBP value and a low GVD characteristics. Under the constant group index criterion [16,40], the normalized frequency bandwidths of $0.444 \%, 0.481 \%, 0.485 \%$, and $0.491 \%$ are obtained for group indices of 77, 68, 64, and 60, respectively. Consequently, the corresponding NDBP are 0.342, 0.327, 0.311 , and 0.295 respectively. This NDBP values are higher than previous works based on modifying the rod radius $[31,42,46]$. The results of our design and previous works are summarized in Table 1. The corresponding GVD characteristics are illustrated in Figure 5b, we find that the GVD parameters $\beta_{2}$ in the slow light region keep around $10^{4}\left(\frac{a}{2 \pi c^{2}}\right)$ for all four cases. This value is two orders of magnitude smaller than the previous works $[43,44]$ and it indicates a more superior performance of our design. In the cases of $r_{1}=0.077 a, r_{2}=0.113 a ; r_{1}=0.079 a, r_{2}=0.117 a$; and $r_{1}=0.08 a, r_{2}=0.118 a$ both negative $\beta_{2}$ values and positive $\beta_{2}$ values are observed, it may reveals potential applications in 
dispersion-compensation. Zero $\beta_{2}$ values is observed in the range of $4.2786 \mathrm{GHz}$ to $4.2925 \mathrm{GHz}$ (the corresponding bandwidth is $0.324 \%$ ) in the case of $r_{1}=0.078 a, r_{2}=0.115 a$. The dispersion of such slow light PCW can be pursued to achieve a much slower $v_{g}$ and lower $\beta_{2}$ values simultaneously by further adjusting the YIG rod radii of the third row adjacent to the waveguide. Notably, when the externally applied magnetic field is reversed, the slope of the dispersion curves have only negative values as shown in Figure $3 \mathrm{~b}$. It indicates that the direction of the group velocity can be controlled by the external magnetic field.

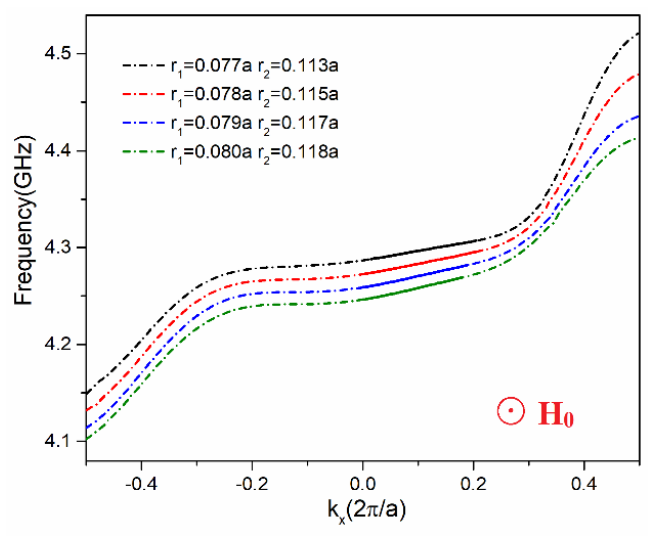

(a)

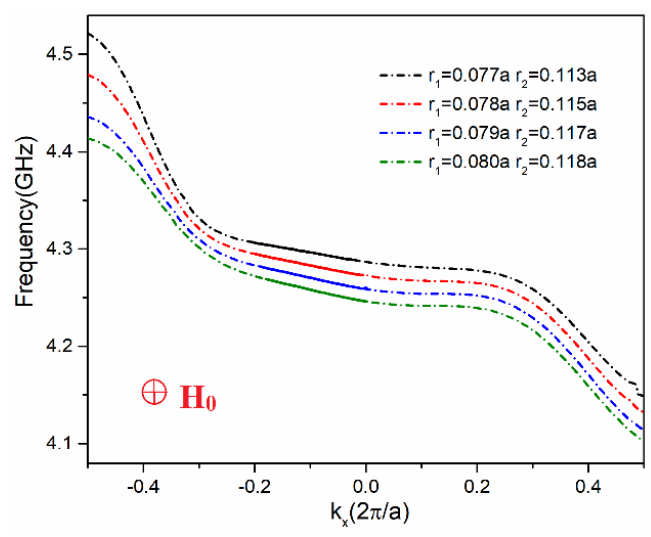

(b)

Figure 4. Dispersion curves of the edge modes for parameters $r_{1}=0.077 a, r_{2}=0.113 a ; r_{1}=0.078 a$, $r_{2}=0.115 a ; r_{1}=0.079 a, r_{2}=0.117 a$; and $r_{1}=0.08 a, r_{2}=0.118 a$. under the $(\mathbf{a})+z$ and $(\mathbf{b})-z$ direction applied magnetic field, The thick solid lines represent the flat band region for slow light. $\odot$ and $\oplus$ indicate $+z$ and $-z$ direction externally applied magnetic field, respectively.

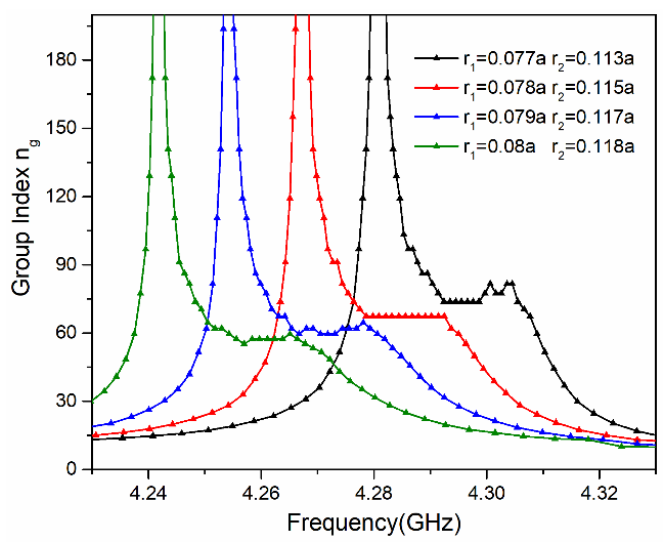

(a)

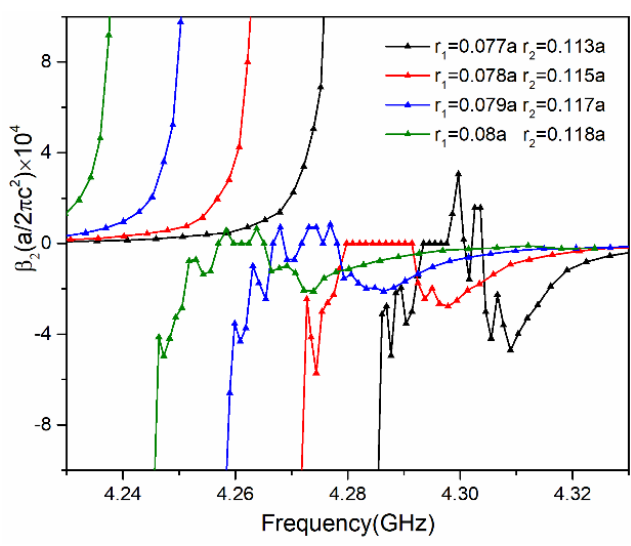

(b)

Figure 5. (a) Calculated group indices $n_{g}$ and (b) the corresponding $\beta_{2}$ as a function of the frequencies for the four optimized PCWs.

The dispersion curve can be tailored further to purse larger NDBP values by adjusting the magnitude of the externally applied magnetic field $H_{0}$. Based on the results of Figure 5, we keep the parameters $r_{1}, r_{2}$ and carry out the optimizations for the $H_{0}$. As shown in Figure 6a, corresponding to the same PCW structures in Figure 5, higher NDBP values of 0.394, 0.347, 0.329, and 0.326 are obtained for $H_{0}=1.277 \times 10^{5} \mathrm{~A} / \mathrm{m}, H_{0}=1.279 \times 10^{5} \mathrm{~A} / \mathrm{m}, H_{0}=1.274 \times 10^{5} \mathrm{~A} / \mathrm{m}$, and $H_{0}=1.277 \times 10^{5} \mathrm{~A} / \mathrm{m}$, respectively. The corresponding GVD characteristics are illustrated in Figure $6 \mathrm{~b}$. It is shown that the GVD parameters $\beta_{2}$ in the slow light region keep around $10^{4}\left(\frac{a}{2 \pi c^{2}}\right)$ for all four cases, and these values are smaller than the values in Figure $5 b$. 


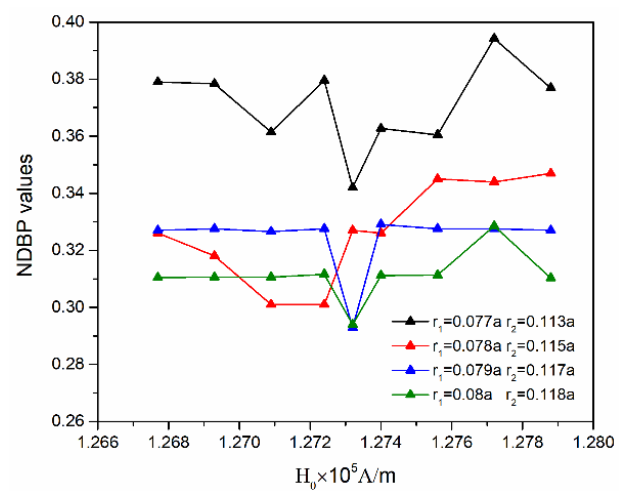

(a)

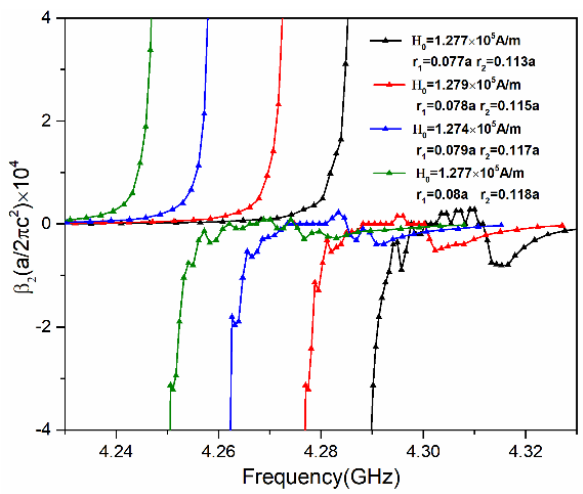

(b)

Figure 6. (a) Normalized delay-bandwidth product (NDBP) values of the four optimized PCWs as a function of the externally applied magnetic field $H_{0} ;(\mathbf{b})$ The $\beta_{2}$ as a function of the frequencies for the four optimized PCWs in Figure 5 with different $H_{0}$.

Table 1. Group index, frequency bandwidth, and NDBP values under different optimized parameters and the comparison with previous works.

\begin{tabular}{ccccc}
\hline Current Work & $\begin{array}{c}\text { Optimized } \\
\text { Parameters }\end{array}$ & $\begin{array}{c}\text { Average Group } \\
\text { Index }\end{array}$ & $\begin{array}{c}\text { Frequency } \\
\text { Bandwidth }\end{array}$ & NDBP Values \\
\hline & $r_{1}=0.077 a$, & 77 & $0.444 \%$ & 0.342 \\
& $r_{2}=0.113 a$ & & & \\
& $r_{1}=0.078 a$, & 68 & $0.481 \%$ & 0.327 \\
& $r_{2}=0.115 a$ & & $0.485 \%$ & 0.311 \\
$r_{1}=0.079 a$, & 64 & $0.491 \%$ & 0.295 \\
& $r_{2}=0.117 a$ & & & \\
$r_{1}=0.08 a$, & 60 & Frequency & NDBP Values \\
\hline \multirow{2}{*}{ Previous Work } & $r_{2}=0.118 a$ & Bverage Group & $0.813 \%$ & 0.186 \\
& Modification of & Index & $0.896 \%$ & 0.233 \\
the Rod Radius & 22.85 & $0.65 \%$ & 0.258 \\
& Ref. [31] & 26 & $0.43 \%$ & 0.268 \\
\hline
\end{tabular}

\subsection{Backscattering-Immune Feature}

As illustrated in Figure 4a, with an external magnetic field applied along the $+z$ direction, the tailored four PCWs still have only positive group velocities (the slope of the waveguide dispersions). It indicates that the waveguide supports only forward transmission and the opposite direction transmission is completely prohibited. The absence of backward propagation channel avoids the possibility of backscattering loss and enables the electromagnetic wave to wrap around different types of obstacles as verified in Figure 7. We introduce four types of interfacial disorders into the waveguide to investigate the robustness of the edge modes. For comparison, Figure 7a shows the $E_{z}$ field distributions without introducing the disorders. As shown in Figure 7b, the light bypasses the inserted metal reflector and maintain the forward transmission. This can never happen in a conventional PCW, as the metal reflector would cause strong scattering and even block the guide mode. However, in the proposed unidirectional PCW, a new interface between the metal reflector and the MOPC is formed, thus providing a way for the electromagnetic wave to flow around the metal reflector and no backscattering takes place. The second type of defect is shown in Figure $7 \mathrm{c}$, when two YIG rods at the interface of the MOPC are replaced by the Copper rods with the same size, the electromagnetic wave flow around the metal rods without introducing backscattering. The third type of defect is a Z-shaped interface, as shown in Figure $7 \mathrm{~d}$, the light travels along the Z-shaped interface 
and the forward propagation is not affected by the two sharp corners. In Figure 7e, two Alumina rods and two YIG rods at the interface of the waveguide (circled in the black rectangles) are replaced by two Alumina rods with smaller rod radii $r_{3}=0.08 a$ and two YIG rods with larger rod radii $r_{4}=0.11 a$, respectively. It is found that the unidirectional transmission is not affected by the interfacial defects. This ideal robust unidirectional transmission feature is of great significance for slow light systems. The same unidirectionality and robustness of the edge modes from all four types of perturbation is observed when the external magnetic field is applied in the $-z$ direction (bottom plots in Figure $7 \mathrm{~b}-\mathrm{e}$ ). The propagation of the electromagnetic wave in the waveguide is switched to the opposite direction accordingly in all five cases.

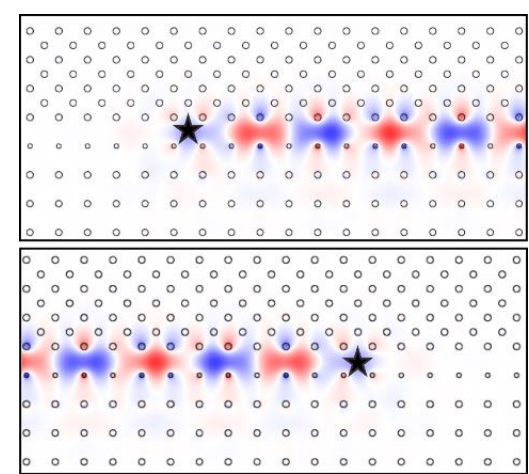

(a)

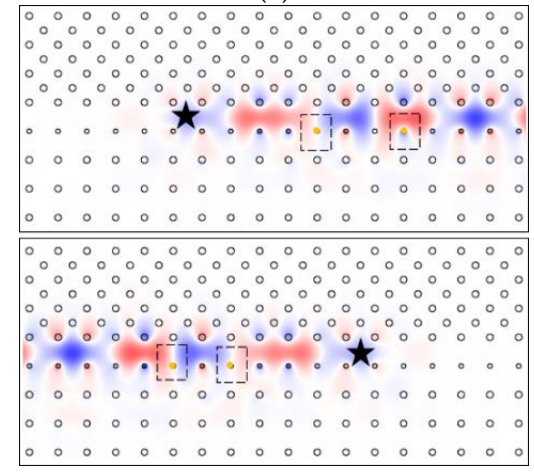

(c)

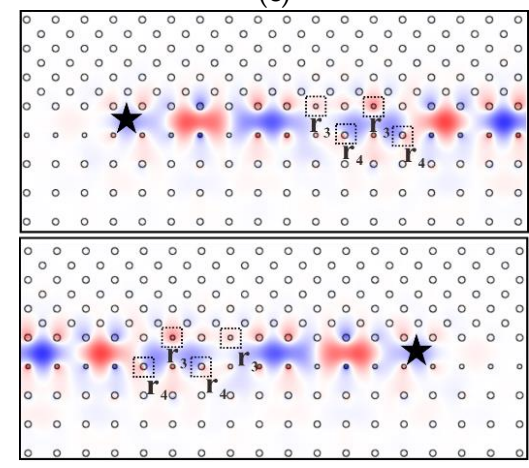

(e)

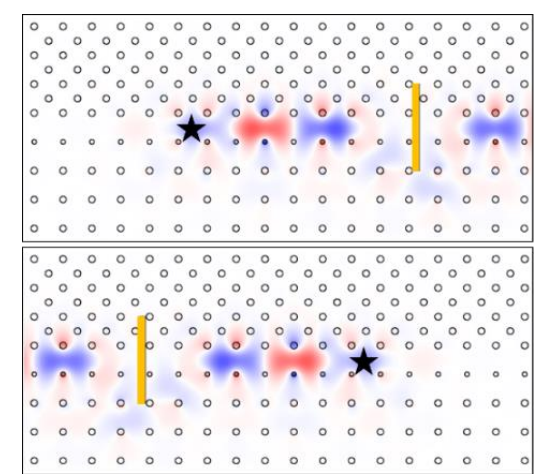

(b)

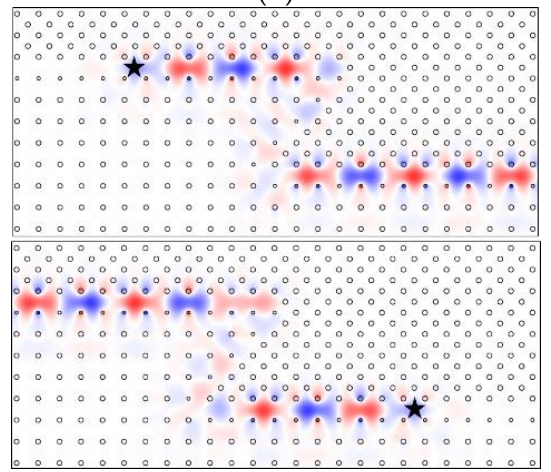

(d)

Figure 7. The electric field distributions for $E_{z}$ at $4.2926 \mathrm{GHz}$ in the unidirectional slow light PCW for parameters $r_{1}=0.078 a, r_{2}=0.115 a$ (a) in the absence of the obstacles; (b) with an inserted metal reflector (indicated by the yellow rectangle); (c) with two YIG rods replaced by Copper rods (circled in the black rectangles) of the same size; (d) with a Z-shaped interface; (e) with rod defects (circled in the black rectangles and the corresponding rod radii are $r_{3}=0.08 a$ and $r_{4}=0.11 a$ respectively). The top and the bottom plots in (a-e) correspond to the results of the externally applied magnetic field along $+z$ and $-z$ direction, respectively. The point source is indicated by the black star. Blue and red colors represent negative and positive field strength values. 
Notably, as illustrated in the top plots of Figure 8, in all five cases, the power flow is cycling anticlockwise on the edge of the Alumina PC and clockwise on the edge of the MOPC. The opposite cycling direction of the power flow on the upper and the lower boundaries of the waveguide core may indicate the slow light properties of the unidirectional waveguide. Under the reversed externally applied magnetic field in the $-z$ direction, the power flow is switched to the opposite direction accordingly as demonstrated in the bottom plots in Figure 8. The results in Figures 7 and 8 are obtained by using a finite-element frequency domain analysis. The point source is located in the interface between two types of PC structures and perfectly matched absorbing boundary layers are applied to the surroundings of the structure in the FEM simulation.

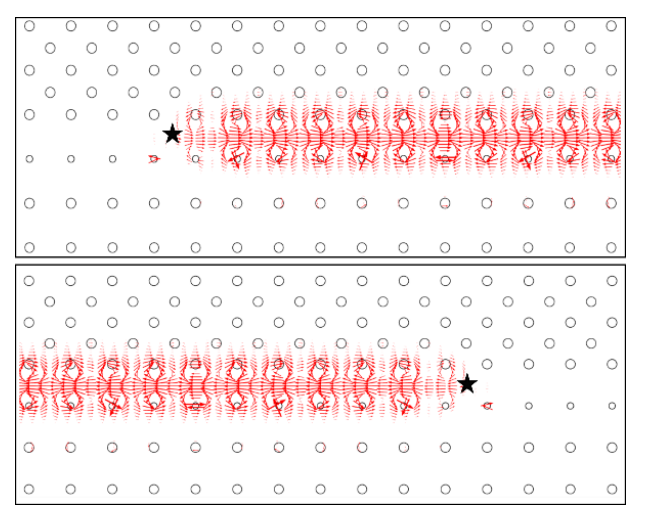

(a)

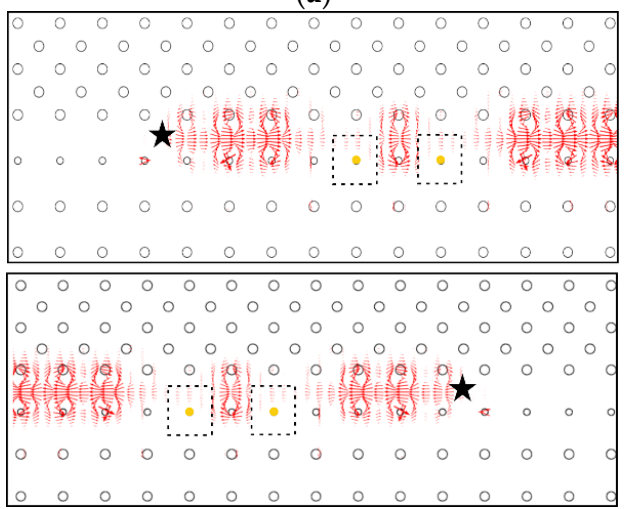

(c)

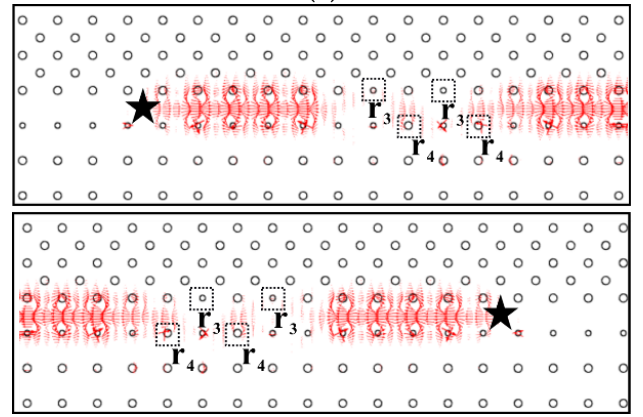

(e)

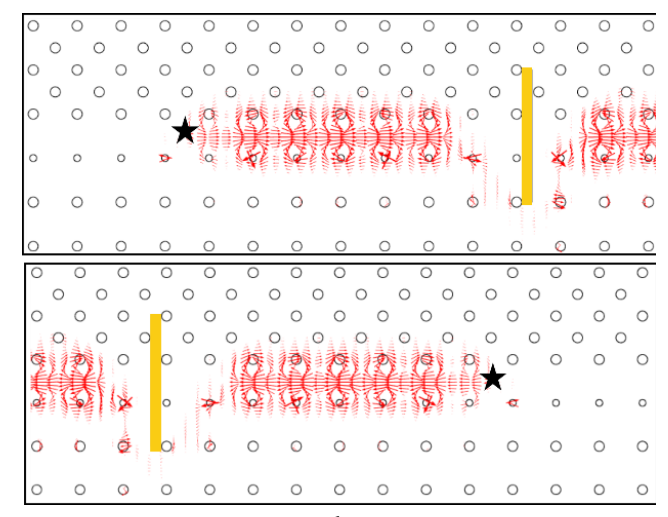

(b)

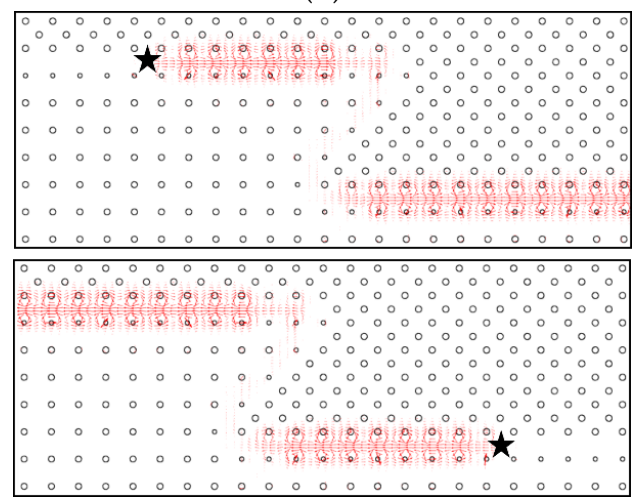

(d)

Figure 8. The Poynting vectors distributions at $4.2926 \mathrm{GHz}$ in the unidirectional slow light PCW for parameters $r_{1}=0.078 a, r_{2}=0.115 a$ (a) in the absence of the obstacles; (b) with an inserted metal reflector (indicated by the yellow rectangle); (c) with two YIG rods replaced by Copper rods (circled in the black rectangles) of the same size; (d) with a Z-shaped interface; (e) with rod defects (circled in the black rectangles and the corresponding rod radii are $r_{3}=0.08 a$ and $r_{4}=0.11 a$ respectively). The top and the bottom plots in (a-e) correspond to the results of the externally applied magnetic field along $+z$ and $-z$ direction, respectively. 


\section{Conclusions}

In this paper, we propose a slow light PCW with unique unidirectionality and robustness characteristics. By choosing appropriate rod radii of the first two rods of the MOPC adjacent to the waveguide core, a high NDBP of 0.342 with a group index of 77 and frequency bandwidth of $0.444 \%$ is obtained. This value is much higher than previously reported works based on radius adjustment. The corresponding GVD parameters $\beta_{2}$ is zero in the range of $4.2786 \mathrm{GHz}$ to 4.2925 GHz. By further adjusting the magnitude of the externally applied magnetic field, much larger NDBP values of 0.394 is obtained. Furthermore, due to the absence of the backward-propagation channel, the backscattering is completely suppressed. Our simulation results show that the edge modes are robust against the introduced interfacial defects. It is of great significance to solving the scattering loss limitations in slow light systems.

Author Contributions: Supervision, X.L.; Writing-Original Draft Preparation, Q.Z.; Writing-Review and Editing, Q.Z.

Funding: This research received no external funding.

Conflicts of Interest: The authors declare no conflict of interest.

\section{References}

1. Thévenaz, L. Slow and fast light in optical fibres. Nat. Mater. 2008, 2, 474-481. [CrossRef]

2. Krauss, T.F. Why do we need slow light. Nat. Photonics 2008, 2, 448-450. [CrossRef]

3. Castellanos Munoz, M.; Petrov, A.Y.; O’Faolain, L.; Li, J.; Krauss, T.F.; Eich, M. Optically induced indirect photonic transitions in a slow light photonic crystal waveguide. Phys. Rev. Lett. 2014, 112, 053904. [CrossRef] [PubMed]

4. Scullion, M.G.; Arita, Y.; Krauss, T.F.; Dholakia, K. Enhancement of optical forces using slow light in a photonic crystal waveguide. Optica 2015, 2, 816. [CrossRef]

5. Yan, S.; Zhu, X.; Frandsen, L.H.; Xiao, S.; Mortensen, N.A.; Dong, J.; Ding, Y. Slow-light-enhanced energy efficiency for graphene microheaters on silicon photonic crystal waveguides. Nat. Commun. 2017, 8, 14411. [CrossRef] [PubMed]

6. Monat, C.; Grillet, C.; Collins, M.; Clark, A.; Schroeder, J.; Xiong, C.; Li, J.; O'Faolain, L.; Krauss, T.F.; Eggleton, B.J.; et al. Integrated optical auto-correlator based on third-harmonic generation in a silicon photonic crystal waveguide. Nat. Commun. 2014, 5, 3246. [CrossRef] [PubMed]

7. Zhao, Y.; Zhang, Y.N.; Qi Wang, M.; Hu, H. Review on the Optimization Methods of Slow Light in Photonic Crystal Waveguide. IEEE Trans. Nanotechnol. 2015, 14, 407-426. [CrossRef]

8. Tamura, T.; Kondo, K.; Terada, Y.; Hinakura, Y.; Ishikura, N.; Baba, T. Silica-Clad Silicon Photonic Crystal Waveguides for Wideband Dispersion-Free Slow Light. J. Lightw. Technol. 2015, 33, 3034-3040. [CrossRef]

9. Serna, S.; Colman, P.; Zhang, W.; Le Roux, X.; Caer, C.; Vivien, L.; Cassan, E. Experimental GVD engineering in slow light slot photonic crystal waveguides. Sci. Rep. 2016, 6, 26956. [CrossRef] [PubMed]

10. Passoni, M.; Gerace, D.; O'Faolain, L.; Andreani, L.C. Optimizing band-edge slow light in silicon-on-insulator waveguide gratings. Opt. Exp. 2018, 26, 8470-8478. [CrossRef] [PubMed]

11. Kondo, K.; Tatebe, T.; Hachuda, S.; Abe, H.; Koyama, F.; Baba, T. Fan-beam steering device using a photonic crystal slow-light waveguide with surface diffraction grating. Opt. Lett. 2017, 42, 4990-4993. [CrossRef] [PubMed]

12. Elshahat, S.; Khan, K.; Yadav, A.; Bibbò, L.; Ouyang, Z. Slow-light transmission with high group index and large normalized delay bandwidth product through successive defect rods on intrinsic photonic crystal waveguide. Opt. Commun. 2018, 418, 73-79. [CrossRef]

13. Ebnali-Heidari, A.; Prokop, C.; Heidari, M.E.; Karnutsch, C. A Proposal for Loss Engineering in Slow-Light Photonic Crystal Waveguides. J. Lightw. Technol. 2015, 33, 1905-1912. [CrossRef]

14. Minkov, M.; Savona, V. Wide-band slow light in compact photonic crystal coupled-cavity waveguides. Optica 2015, 2, 631-634. [CrossRef]

15. Schulz, S.A.; Upham, J.; O’Faolain, L.; Boyd, R.W. Photonic crystal slow light waveguides in a kagome lattice. Opt. Lett. 2017, 42, 3243-3246. [CrossRef] [PubMed] 
16. Tang, J.; Wang, T.; Li, X.; Wang, B.; Dong, C.; Gao, L.; Liu, B.; He, Y.; Yan, W. Wideband and Low Dispersion Slow Light in Lattice-Shifted Photonic Crystal Waveguides. J. Lightw. Technol. 2013, 31, 3188-3194. [CrossRef]

17. Johnson, S.G.; Povinelli, M.L.; Soljaci, M.; Karalis, A.; Jacobs, S.; Joannopoulos, J.D. Roughness losses and volume-current methods in photonic-crystal waveguides. Appl. Phys. B 2005, 81, 283-293. [CrossRef]

18. Povinelli, M.L.; Johnson, S.G.; Lidorikis, E.; Joannopoulos, J.D.; Soljačić, M. Effect of a photonic band gap on scattering from waveguide disorder. Appl. Phys. Lett. 2004, 84, 3639-3641. [CrossRef]

19. Hughes, S.; Ramunno, L.; Young, J.F.; Sipe, J.E. Extrinsic optical scattering loss in photonic crystal waveguides: Role of fabrication disorder and photon group velocity. Phys. Rev. Lett. 2005, 94, 033903. [CrossRef] [PubMed]

20. Kuramochi, E.; Notomi, M.; Hughes, S.; Shinya, A.; Watanabe, T.; Ramunno, L. Disorder-induced scattering loss of line-defect waveguides in photonic crystal slabs. Phys. Rev. B 2005, 72. [CrossRef]

21. Ao, X.; Lin, Z.; Chan, C.T. One-way edge mode in a magneto-optical honeycomb photonic crystal. Phys. Rev. B 2009, 80, 033105. [CrossRef]

22. He, C.; Sun, X.C.; Liu, X.P.; Lu, M.H.; Chen, Y.; Feng, L.; Chen, Y.F. Photonic topological insulator with broken time-reversal symmetry. Proc. Natl. Acad. Sci. USA 2016, 113, 4924-4928. [CrossRef] [PubMed]

23. Khanikaev, A.B.; Mousavi, S.H.; Tse, W.-K.; Kargarian, M.; MacDonald, A.H.; Shvets, G. Photonic topological insulators. Nat. Mater. 2013, 12. [CrossRef] [PubMed]

24. Lu, L.; Joannopoulos, J.D.; Soljačić, M. Topological Photonics. Nat. Photonics 2014, 8, 821-829. [CrossRef]

25. Haldane, F.D.M.; Raghu, S. Possible realization of directional optical waveguides in photonic crystals with broken time-reversal symmetry. Phys. Rev. Lett. 2008, 100, 013904. [CrossRef] [PubMed]

26. Raghu, S.; Haldane, F.D.M. Analogs of quantum-Hall-effect edge states in photonic crystals. Phys. Rev. A 2008, 78. [CrossRef]

27. Wang, Z.; Chong, Y.; Joannopoulos, J.D.; Soljacic, M. Observation of unidirectional backscattering-immune topological electromagnetic states. Nature 2009, 461, 772-775. [CrossRef] [PubMed]

28. Wang, Z.; Chong, Y.D.; Joannopoulos, J.D.; Soljacic, M. Reflection-free one-way edge modes in a gyromagnetic photonic crystal. Phys. Rev. Lett. 2008, 100, 013905. [CrossRef] [PubMed]

29. Poo, Y.; Wu, R.X.; Lin, Z.; Yang, Y.; Chan, C.T. Experimental realization of self-guiding unidirectional electromagnetic edge states. Phys. Rev. Lett. 2011, 106, 093903. [CrossRef] [PubMed]

30. Hafezi, M.; Demler, E.A.; Lukin, M.D.; Taylor, J.M. Robust optical delay lines with topological protection. Nat. Phys. 2011, 7, 907-912. [CrossRef]

31. Kurt, H.; Üstün, K.; Ayas, L. Study of different spectral regions and delay bandwidth relation in slow light photonic crystal waveguides. Opt. Exp. 2010, 18, 26965-26977. [CrossRef] [PubMed]

32. Liang, J.; Ren, L.Y.; Yun, M.J.; Han, X.; Wang, X.J. Wideband ultraflat slow light with large group index in a W1 photonic crystal waveguide. J. Appl. Phys. 2011, 110, 063103. [CrossRef]

33. Pourmand, M.; Karimkhani, A.; Nazari, F. Wideband and low-dispersion engineered slow light using liquid infiltration of a modified photonic crystal waveguide. Appl. Opt. 2016, 55, 10060-10066. [CrossRef] [PubMed]

34. Lü, S.; Zhao, J.; Zhang, D. Flat band slow light in asymmetric photonic crystal waveguide based on microfluidic infiltration. Appl. Opt. 2010, 49, 3930-3934. [CrossRef] [PubMed]

35. Ebnali-Heidari, M.; Grillet, C.; Monat, C.; Eggleton, B.J. Dispersion engineering of slow light photonic crystal waveguides using microfluidic infiltration. Opt. Exp. 2009, 17, 1628-1635. [CrossRef]

36. Nazari, F.; Samsami Khodadad, F. On chip optical isolator based on non-linear silicon photonic crystal by using asymmetric engineering waveguide. J. Mod. Opt. 2017, 64, 653-658. [CrossRef]

37. Üstün, K.; Kurt, H. Ultra slow light achievement in photonic crystals by merging coupled cavities with waveguides. Opt. Exp. 2010, 20, 21155-21161. [CrossRef] [PubMed]

38. Xu, Y.; Xiang, L.; Cassan, E.; Gao, D.; Zhang, X. Slow light in an alternative row of ellipse-hole photonic crystal waveguide. Appl. Opt. 2013, 52, 1155-1160. [CrossRef] [PubMed]

39. Hao, R.; Cassan, E.; Kurt, H.; Roux, X.L.; Marris-Morini, D.; Vivien, L.; Wu, H.; Zhou, Z.; Zhang, X. Novel slow light waveguide with controllable delay-bandwidth product and utra-low dispersion. Opt. Exp. 2010, 18, 5492-5950. [CrossRef] [PubMed]

40. Petrov, A.Y.; Eich, M. Zero dispersion at small group velocities in photonic crystal waveguides. Appl. Phys. Lett. 2004, 85, 4866-4868. [CrossRef]

41. Lenz, G.; Eggleton, B.J.; Madsen, C.K.; Slusher, R.E. Optical Delay Lines Based on Optical Filters. IEEE J. Quantum Electron. 2001, 37, 525-532. [CrossRef] 
42. Frandsen, L.H.; Lavrinenko, A.V.; Fage-Pedersen, J.; Borel, P.I. Photonic crystal waveguides with semi-slow light and tailored dispersion properties. Opt. Exp. 2006, 14, 9444-9450. [CrossRef]

43. Engelen, R.J.P.; Sugimoto, Y.; Watanabe, Y.; Korterik, J.P.; Ikeda, N.; Hulst, N.F.; Asakawa, K.; Kuipers, L. The effect of higher-order dispersion on slow light propagation in photonic crystal waveguides. Opt. Exp. 2006, 14, 1658-1672. [CrossRef]

44. Ma, J.; Jiang, C. Demonstration of Ultraslow Modes in Asymmetric Line-Defect Photonic Crystal Waveguides. IEEE Photonics Technol. Lett. 2008, 20, 1237-1239. [CrossRef]

45. Pozar, D.M. Microwave Engineering, 4st ed.; Wiley: New York, NY, USA, 2012; pp. 452-458. ISBN 978-0-47063155-3.

46. Hou, J.; Gao, D.; Wu, H.; Hao, R.; Zhou, Z. Flat Band Slow Light in Symmetric Line Defect Photonic Crystal Waveguides. IEEE Photonics Technol. Lett. 2009, 21, 1571-1573. [CrossRef]

47. Aghababaeian, H.; Vadjed-Samiei, M.H.; Granpayeh, N. Temperature Stabilization of Group Index in Silicon Slotted Photonic Crystal Waveguides. J. Opt. Soc. Korea 2011, 15, 398-402. [CrossRef]

48. Tian, H.; Zhou, J.; Zhai, Y.; Ji, Y. Flat Band Slow Light Performance in Dual-Slot Silicon-on-Insulator Based Photonic Crystal Waveguide. Jpn. J. Appl. Phys. 2013, 52, 032001. [CrossRef]

(C) 2018 by the authors. Licensee MDPI, Basel, Switzerland. This article is an open access article distributed under the terms and conditions of the Creative Commons Attribution (CC BY) license (http://creativecommons.org/licenses/by/4.0/). 